\title{
Event-switched control design with guaranteed performances
}

\author{
B. Boisseau ${ }^{1 *}$, J.J. Martinez ${ }^{1}$, T. Raharijaona ${ }^{2}$, S. Durand ${ }^{3}$, N. Marchand ${ }^{1}$ \\ ${ }^{1}$ Univ. Grenoble Alpes, GIPSA-Lab, F-38000 Grenoble, France \\ CNRS, GIPSA-Lab, F-38000 Grenoble, France \\ ${ }^{2}$ Aix-Marseille Univ., ISM UMR 7287, 13288, Marseille cedex 09, France \\ ${ }^{3}$ INSA Strasbourg, ICube, Univ. Strasbourg, CNRS, Strasbourg, France
}

\begin{abstract}
SUMMARY
In this paper, a new event-switched control method is presented for controlling discrete-time linear systems subject to bounded disturbances. The main advantage of the proposed method is that the nominal performance of the controlled system with periodic control updates is kept in a framework that do not require to periodically update the control law. The feedback control loop can be opened as long a state-dependent event condition is satisfied. This condition is obtained using set theory approaches. In particular, the concept of robustly positively invariant sets is used to calculate the nominal performance and the event condition. The simulation presented in this paper confirms the efficiency of the present approach. A reduction of the numerical complexity of the approach is also proposed. Copyright (c) 0000 John Wiley \& Sons, Ltd.
\end{abstract}

Received ...

KEY WORDS: event-based; event-switched; event-triggered; positive invariance; robust control; perturbed linear systems

\section{INTRODUCTION}

Classical control theory can be used to design continuous and discrete-time controllers. Nowadays, discrete controllers are being increasingly used because digital technology is supplanting analog solutions, apart from a few exceptions involving particularly stringent constraints. Most of these digital technologies depend on the use of a fixed clock frequency. In this framework, many wellknown methods have been developed for designing controllers (see for instance [1]). Unfortunately, although periodicity often simplifies the design, it results in a conservative resource management [2]. This is especially true in the case of continuous time nonlinear systems, where no discretized model for the system is usually available. Discrete time control therefore often consists in designing the controller in the continuous time domain and updating the control value with a sufficiently high sampling rate. However, the hardware used to sample and hold the output of the system or to compute the feedback control loop is liable to be incompatible with having a sufficiently small sampling period to obtain acceptable closed-loop performances, as previously reported in [3]. Solutions such as sampled-data control algorithms have also been developed, based on an approximation for the discrete time model [4] or a redesigned continuous time controller [5]. However, all these methods are relatively complex and difficult to implement.

The relevance of periodic sampling methods has often been questioned during the last few decades, and event-based control approaches have been suggested as an alternative solution. With classical time-triggered approaches, control law is computed and the control signal is updated

${ }^{*}$ Correspondence to: Bruno Boisseau, GIPSA-Lab, France. E-mail: bruno.boisseau@ gipsa-lab.fr 
at the same rate whether this is really necessary or not, whereas event-based procedures do not require these periodic computations and updates, but calls for resources only when they are strictly necessary. When the system variables undergo no significant changes, the sensors' sampling rate and control actuation should be kept to a minimum. Event-based control design consists not only in determining how to actuate the system, but also when to actuate it. An event function is therefore used to determine the instants, which are called events, when the control law has to be updated (to ensure satisfactory performances or stability, for instance), and the control signal is otherwise kept constant between two successive events. In [6] for example, the problem of limiting the number of control updates was addressed for the first time in the case of a simple PID controller. The idea consisted here of monitoring the activity of the system in order to compute the control law and update the control signal only when the dynamics of the system crossed a given threshold (this is known as level-crossing sampling). Event-based control is clearly an opportunity for enhancing embedded and networked control systems with limited resources. However, the implementation of event-based control strategies can result in additional challenges, such as determining how frequently the control signal has to be updated and applied so as to ensure that the performances and the stability of the system are not affected.

Although event-based control systems are obviously a great step forward, very few theoretical studies have dealt with aspects such as the stability, convergence and performances of this approach. Event-detection processes have been taken to depend on changes in the state (or at least in the output) of the system, as in [6-14]. It has been established in [10] in particular that the control law can be updated less frequently than with a periodic scheme, while still giving equally satisfactory performances. Stabilization of linear and nonlinear and even PDE systems has been analysed in [15-21], where the events are given by changes in a Lyapunov function or the time derivative of a Lyapunov function (and consequently also in the state). Event-based scheme has also been proposed for multi-agents systems to achieve a consensus while avoiding unnecessary communication and control update, as in [22-26]. Another important issue for event-based control theory is the Zeno phenomenon (where an infinite number of events occur during a finite length of time). This behavior has to be avoided in practice, which is generally done by searching for a guaranteed minimum sampling interval. This is not necessary, however, when implementing eventbased control methods on digital platforms because the control process is updated up to a certain condition, which is checked periodically: the minimum sampling interval can therefore not be smaller than the sampling period. This method has been classified as Periodic Event-Triggered Control in [27], an approach which strikes a balance between conventional periodic sampleddata control and event-triggered control. Note that this approach is often unsuitable for use with nonlinear systems, where the appropriate period can sometimes depend on the initial condition [18]. Existing event-based methods often involve making a trade-off between the number of control updates and the performances of the system. In other words, savings in terms of computational and/or communication resources can result in less satisfactory closed-loop performances (but not always, see [18]). Asymptotic stability of an event-triggered system is ensured here by including the existence of a Lyapunov function.

However, in the case of uncertain linear discrete-time dynamic systems subjected to bounded disturbances, asymptotic stability cannot be ensured. Practical stability, which has been discussed by LaSalle and Lefschetz in [28], is the best that can be achieved in this case. Although some studies have focused on the most suitable sampling schemes for first order stochastic systems (see for example $[2,10,29]$ ), very few authors have investigated this topic in the case of higher order perturbed systems. In this context, the results obtained on ultimate boundedness and invariant sets are suitable for use in stability analysis. If a time-triggered closed-loop system without any disturbances is stable, a Robustly Positively Invariant (RPI) set can be taken to exist for the perturbed system. This set describes a region of the state space where the state of the closed-loop system subjected to bounded disturbances will continue to stay in this set. In addition, ultimate boundedness (UB) of the event-switched system to a bounded set can also be assumed to exist, which means that with any initial condition, the state will reach a set within a finite time and continue to stay in this set. 
These properties are closely related to the topic of practical stability. Readers are referred to [30,31] for the theory and applications of ultimate bounds and RPI sets.

Very few studies have been published in which event-based control theory has been combined with set theory. Heemels et al. have introduced an event-triggering process giving bounded ultimate bounds in the case of perturbed linear systems. In [32] the authors proposed an event-based control based on set theory where a RPI set in guaranteed for the event-based system. However, with this approach, the same minimal Robustly Positively Invariant (mRPI) set (the RPI set included in all possible RPI sets) cannot be guaranteed for both time-triggered and event-triggered systems. In other words, with the methods mentioned above, a trade-off is necessary between the number of control updates and the performances of the system.

In the present paper, a systematic method is presented for designing an event-switched mechanism, which gives the same mRPI set in the case of both time-triggered and event-switched systems. The other existing methods consist in designing the event mechanism and computing the performances a posteriori, whereas the present method makes it possible to specify the performances required (in terms of the RPI set targeted) a priori and to compute the event condition accordingly. Another difference in comparison with previous studies on event-based control is that we do not determine when a control law has to be updated, but only when we have to switch from one control law to another (which may render the system unstable). More specifically, we focus on switching between a closed-loop control law and a constant control law (and vice versa). For the sake of simplicity, the closed-loop control is updated periodically in the present paper but it could also be event-based. The proposed approach is therefore complementary to event-based control. As event-based control, the event switched control enables to save communication bandwidth in the context of a networked controlled system, since no communication between the controller and the actuators is required as long as the control input is kept constant. Another interest of the proposed approach that event-based control does not provide concerns safe control of system by humans. In that case, the constant control mode will correspond to the human controlled mode. The action of the human operator is considered as a perturbation to the system. When his action fails to achieve a given performance, the system switches to the closed-loop mode to bring the system back into an acceptable configuration.

The rest of this paper is organized as follows. In Section 1, definitions are presented and the problems it is proposed to investigate are formulated. Some preliminary considerations about invariant sets are recalled and the method used to compute the event condition is introduced in Section 2. A useful means of reducing the complexity of the event-set is also provided for implementation purposes. The main contributions of this study are then described in detail in Section 3. In particular, the main results are presented in Section 3.1, the performances of the eventswitching strategy are assessed in Section 3.2, a solution with an observer is suggested in Section 3.3 for use in cases where complete state information is not available, and the resulting algorithm corresponding to the whole solution proposed is given in Section 3.4. Results of simulations are presented and discussed in Section 4 in the case of a second order system. Lastly, our conclusions are presented and possible lines of future research are suggested in Section 5.

\section{PROBLEM STATEMENT}

Let us take the following discrete-time linear time-invariant system:

$$
x_{k+1}=A x_{k}+B u_{k}+E w_{k}
$$

where $A \in \mathbb{R}^{n \times n}, B \in \mathbb{R}^{n \times m}, E \in \mathbb{R}^{n \times f}$, and $k \in \mathbb{N}$. $x_{k} \in \mathbb{R}^{n}$ denotes the current state of the system, $x_{k+1}$ is the successor state, $u_{k} \in \mathbb{R}^{m}$ denotes the control input, and $w_{k} \in \mathcal{W}$ denotes the unknown bounded disturbances. $\mathcal{W}$ is assumed to be a compact convex polytope in $\mathbb{R}^{f}$ defined by $p$ vertices $\mathcal{W}_{i}$ as follows: $\mathcal{W} \triangleq \operatorname{conv}\left\{\mathcal{W}_{i}\right\}$ for $i=\{1, \ldots, p\}$. The set $\mathcal{W}$ is taken to include the origin, and $\operatorname{rank}(E)$ is assumed to be greater than or equal to $n$ : the set $E \mathcal{W}$ therefore also includes the origin. It is also assumed that the matrix pair $(A, B)$ is stabilizable using the linear discrete-time 
state feedback:

$$
u_{k}=-K x_{k}
$$

where $K \in \mathbb{R}^{n \times m}$ is the state-feedback matrix. The dynamics of the closed-loop system can therefore be described by the following equation:

$$
x_{k+1}=\underbrace{(A-B K)}_{A_{C L}} x_{k}+E w_{k}
$$

where all the eigenvalues of the closed-loop matrix $A_{C L}$ are located inside the unit circle. In what follows, the system dynamics (3) will be referred to as the time-triggered system. Before defining the event-switched system, which is the most original aspect of the present paper, some additional definitions will be recalled:

Definition 1 (From [31])

A set $\mathcal{S} \subseteq \mathbb{R}^{n}$ is said to be a Robustly Positively Invariant (RPI) set for a system $x_{k+1}=f\left(x_{k}, w_{k}\right)$, if for any initial conditions $x_{0}$ in $\mathcal{S}$ and any disturbances $w_{k}$ in $\mathcal{W}, x_{k}$ will belong to $\mathcal{S}$ for all instants $k \in \mathbb{N}^{+}$.

Definition 2 (From [30])

A system $x_{k+1}=f\left(x_{k}, w_{k}\right)$ is said to be Uniformly Ultimately Bounded (UB) to the set $\mathcal{S}$ if for all initial conditions $x_{0} \in \mathbb{R}^{n}$ and any disturbances $w_{k}$ in $\mathcal{W}$, there exists a positive instant $T\left(x_{0}\right)$ such that $x_{k}$ remains in $\mathcal{S}$ for all $k \geq T\left(x_{0}\right)$.

Definition 3 (From [33])

A set $\mathcal{S}$ is said to be the minimal Robustly Positively Invariant (mRPI) set for a system $x_{k+1}=$ $f\left(x_{k}, w_{k}\right)$ if $\mathcal{S}$ is a RPI set for this system, and if $\mathcal{S}$ is included in all possible closed RPI sets for this system.

Let $\mathcal{S}_{2}$ be some set in $\mathbb{R}^{n}$. Let us now define the following event-switched control law:

$$
u_{k}= \begin{cases}0_{m} & \text { if } x_{k} \in \mathcal{S}_{2} \\ -K x_{k} & \text { otherwise }\end{cases}
$$

where $0_{m}$ denotes the $m$-dimensional null vector. In the sequel, an event-switched system will refer to a system of the form (1) with a control input as in (4). It is given by the following closed-loop equation:

$$
x_{k+1}= \begin{cases}A x_{k}+E w_{k} & \text { if } x_{k} \in \mathcal{S}_{2} \\ (A-B K) x_{k}+E w_{k} & \text { otherwise }\end{cases}
$$

\section{Remark 1}

For the sake of simplicity, the case of stabilization around the origin will be studied here. In the general case, the control law will also depend on the a priori control input required $u_{e q}$ and the state vector required $x_{e q}$ at equilibrium, for instance:

$$
\bar{u}_{k}=u_{e q}-K\left(x_{k}-x_{e q}\right)
$$

and the event-switched control would be:

$$
\bar{u}_{k}= \begin{cases}u_{e q} & \text { if } x_{k} \in \mathcal{S}_{2} \\ u_{e q}-K\left(x_{k}-x_{e q}\right) & \text { otherwise }\end{cases}
$$

The signals $u_{e q}$ and $x_{e q}$ can be computed at the plant level at all times. These signals can be desired path-planning or reference model signals, for example.

Problem statement: Given the plant model (1), a state feedback (2) and a RPI set $\mathcal{S}_{1}$ for the timetriggered state-feedback loop (3), find an event condition (i.e. a condition based on a set $\mathcal{S}_{2}$ ) and an 
event-switched mechanism (4) such that the set $\mathcal{S}_{1}$ is a RPI set for both the time-triggered system (3) and the event-switched system (5).

In this paper, the event-switched mechanism is assumed to be given a priori. The aim is to set the event condition giving the event-triggered system (5) the same performances as the time-triggered system (3) (in terms of RPI set). The event condition makes the mechanism switch from the open loop system to the system controlled by a state feedback law.

In what follows, the performances are given by the following four indicators:

- The bound $\delta_{x}$ on the 2-norm of the state $x$;

- The bound $\delta_{u}$ on the 2-norm of the control input $u$;

- The bound $\delta_{d}$ on the 2-norm of the changing rate of the control input: $\Delta u=u_{k+1}-u_{k}$;

- The number of communications required between the controller and the actuator.

The first index will reflect the ability of the controller to stabilize the system, the second one will indicate the cost in terms of the control power, the third one will reflect the changes in the control input, which are usually to the aging of the actuator, and the fourth one will denote the cost in terms of the communication between the controller block and the actuator.

Notation: Given two sets $\mathcal{A} \subset \mathbb{R}^{n}$ and $\mathcal{B} \subset \mathbb{R}^{n}$, the relative complement of $\mathcal{A}$ in $\mathcal{B}$, which will be denoted $\mathcal{B} \backslash \mathcal{A}$, is defined as follows: $\mathcal{B} \backslash \mathcal{A}=\{x \in \mathcal{B} \mid x \notin \mathcal{A}\}$. The Minkowski sum of $\mathcal{A}$ and $\mathcal{B}$, which will be denoted $\mathcal{A} \oplus \mathcal{B}$, is defined as follows: $\mathcal{A} \oplus \mathcal{B}=\{a+b \mid a \in \mathcal{A}, b \in \mathcal{B}\}$.

\section{NOMINAL MINIMAL RPI SET AND EVENT-SET COMPUTATION}

The concept of RPI sets (see $[31,34]$ ) is often used to characterize the nominal performances of the time-triggered system (3). The problem here is how to find an approximation for the mRPI set describing all possible trajectories of the system's state, starting at the origin (in the case of null stabilization) in the presence of bounded disturbances. $\mathcal{S}_{1}$ will denote the nominal performance set of system (3). Since no means of computing an exact mRPI have yet been found when the system's dynamics are not nilpotent, a polyhedral approximation for the mRPI set will be used to characterize what has been called the nominal performance of the time-triggered system.

\subsection{Polyhedral RPI sets for characterizing nominal performances}

Polyhedral RPI sets for linear systems (3) can be computed using methods such as those developed in [35], [36] and [37]. These methods are very useful for computing RPI sets in polyhedral form, especially in the case of stable systems where the eigenvalues of the matrix $A_{C L}$ are real values. A systematic method of obtaining polyhedral approximations for the minimal RPI set is presented in [33]. Polyhedral invariant set computations have many advantages over traditional ultimate-bound methods of computation based on the use of quadratic Lyapunov functions, as discussed in [38] and [39]. In particular, these methods may be less conservative (i.e., they give smaller sets) than ellipsoids describing quadratic Lyapunov functions.

A refined polyhedral approximation for the $\mathrm{mRPI}$ set can be obtained by implementing a sequence of outer approximations of the mRPI set, as described in [40] and [39]. This method consists in building a sequence of RPI sets recursively, based on the Minkowski sum between the image of a RPI set given by the linear transformation $A_{C L}$ and the polyhedral set $E \mathcal{W}$. This gives:

$$
\Phi(s+1)=A_{C L} \Phi(s) \oplus E \mathcal{W}, \quad \Phi(0)=\Psi
$$

where $A_{C L}$ is a stable matrix (all its eigenvalues are located inside the unit circle) and $E \mathcal{W}$ is a polyhedral set which includes all the disturbance trajectories. $\Phi(s)$ is the linear transformation. $\Psi \in \mathbb{R}^{n}$ is a polyhedral describing an initial RPI set estimation of the state trajectories, which can be computed using the method presented in [39]. Therefore, [40] states that for any $\delta>0$, there exists $s^{*} \in \mathbb{N}$ such that the following relation is true:

$$
\Omega_{\infty} \subseteq \Phi\left(s^{*}+1\right) \subseteq \Phi\left(s^{*}\right) \subseteq \Psi
$$


where $\Omega_{\infty}$ denotes the exact mRPI set. Then, $\Phi\left(s^{*}\right) \rightarrow \Omega_{\infty}$ as $s^{*} \rightarrow \infty$, that is:

$$
\Omega_{\infty} \subseteq \Phi\left(s^{*}\right) \subseteq \Omega_{\infty} \oplus \mathbb{B}_{p}^{n}(\varepsilon)
$$

where $\mathbb{B}_{p}^{n}(\varepsilon)$ denotes a $n$-dimensional ball with the radius $\varepsilon$ with respect to the $p$-norm.

Remark 2

The initial set $\Psi$ in the set recursion (6) can in fact be any RPI set for system (3).

Using these approximations, a nominal set $\mathcal{S}_{1}=\Phi\left(s^{*}\right)$ can be obtained by performing a given number of iterations $s^{*} \in \mathbb{N}$ characterizing the targeted accuracy of the approximation for the mRPI set.

\subsection{Event-set computation}

Given a RPI set approximation $\mathcal{S}_{1}$ for system (3), the aim is now to compute a set $\mathcal{S}$ such that:

$$
x_{k} \in \mathcal{S} \Longrightarrow x_{k+1} \in \mathcal{S}_{1} \text {, with } x_{k+1}=A x_{k}+E w_{k}
$$

Definition 4

A set $\mathcal{S}$ is said to be an event-set for system (1) if and only if it satisfies condition (9) for a given set $\mathcal{S}_{1}$.

The following method was developed here for computing the set of maximum volumes meeting (9), denoted $\mathcal{S}_{2}^{\star}$. That is, $\mathcal{S}_{2}^{\star}$ contains all the possible sets which also satisfy the condition (9).

The set $\mathcal{S}_{1}$ is taken to be a polyhedral set with a given half-space representation, that is:

$$
\mathcal{S}_{1} \triangleq\left\{x \in \mathbb{R}^{n}: H x \leq P\right\}
$$

where $H \in \mathbb{R}^{l \times n}$ and $P \in \mathbb{R}^{l}$.

Condition (9) can be translated into a family of inequalities which can be used to find all the possible values of $x_{k}$, via the matrices $\bar{H}$ and $\bar{P}_{i}$, satisfying the following condition:

$$
\begin{aligned}
x_{k+1} \in \mathcal{S}_{1} \Leftrightarrow & H x_{k+1} \leq P \\
& H\left(A x_{k}+E W_{i}\right) \leq P \\
& \underbrace{H A}_{\bar{H}} x_{k} \leq \underbrace{P-H E W_{i}}_{\bar{P}_{i}}
\end{aligned}
$$

where $W_{i}$ denotes the $i^{\text {th }}$ vertex of the polyhedron $\mathcal{W}$.

Therefore, by defining the set:

$$
\mathcal{S}_{2}^{\star} \triangleq\left\{x \in \mathbb{R}^{n}: \bar{H} x \leq \bar{P}_{i}, \text { for } i=\{1, \ldots, p\}\right\}
$$

Then the set $\mathcal{S}_{2}^{\star}$ will include all the event-sets.

$$
x_{k} \in \mathcal{S}_{2}^{\star} \Longrightarrow x_{k+1} \in \mathcal{S}_{1}, \text { with } x_{k+1}=A x_{k}+E w_{k}
$$

This is possible because $\mathcal{S}_{1}$ and $\mathcal{W}$ are convex and compact sets including the origin.

\section{Remark 3}

Please note that:

- $\mathcal{S}_{2}^{\star}$ is a convex set because it can be described by the intersection between a finite number of hyperplanes in (14).

- $\mathcal{S}_{2}^{\star}$ is possibly empty if the inequalities in (14) have no solution.

- $\mathcal{S}_{2}^{\star}$ is not necessarily included in $\mathcal{S}_{1}$. In this particular case, $\mathcal{S}_{2}^{\star} \backslash \mathcal{S}_{1}$ can be reached one time step before entering $\mathcal{S}_{1}$.

- Any set included in $\mathcal{S}_{2}^{\star}$ is an event-set for the system (1) and the set $\mathcal{S}_{1}$. 


\subsection{Reducing the complexity of the event-set}

In order to apply the event-switched control (4), it is necessary to verify at each sampling time if the state of the system is inside the set $\mathcal{S}_{2}$. If this set is polyhedral, then the computational complexity to check the inclusion condition, $x_{k} \in \mathcal{S}_{2}$, depends on the order of the system and the number of hyperplanes describing the set.

An alternative strategy might be to use an ellipsoidal set. This allows to check a simpler inclusion condition, $x_{k} \in \mathcal{S}_{2}$, where:

$$
\mathcal{S}_{2} \triangleq\left\{x \in \mathbb{R}^{n}: x^{T} M x \leq c\right\}
$$

where $M \in \mathbb{R}^{n \times n}$, and $c \in \mathbb{R}$.

The advantage of using an ellipsoidal set is that the complexity of the inclusion condition's evaluation only depends on the order $n$ of the system, whereas a polyhedral set can be described by an arbitrary number of hyperplanes.

\section{EVENT-SWITCHED CONTROL DESIGN WITH GUARANTEED PERFORMANCES}

\subsection{Main results}

Since stabilization at a point for the time-triggered system (1) without perturbation is considered, we assume the very classical property for the pair $(A, B)$ :

\section{Assumption 1}

The pair $(A, B)$ of system (1) is stabilizable.

Let us state three Assumptions that enable to guaranty that if there is a robustly positively invariant set for the time-triggered system, it will still be a robustly positively invariant set in the proposed event-switched framework. In other words, with the proposed event-switched control approach, the system will remain in the same RPI set as the time-triggered system if it starts in it. In addition we also want to guaranty that the property of ultimate boundedness is kept for the event-switched system. This will ensure that for any initial condition, the state of the event-triggered system joins the RPI set in finite number of time steps. For this, the following assumptions are sufficient:

\section{Assumption 2}

The set $\mathcal{S}_{2}$ is an event-set for system (1) and a given set $\mathcal{S}_{1}$.

\section{Assumption 3}

$\mathcal{S}_{1}$ is a RPI set for system (1) with the stabilizing control input (2) and unknown bounded disturbances $w_{k}$.

\section{Assumption 4}

System (1) subjected to the stabilizing control (2) and bounded disturbances $w_{k}$ is UB to the set $\mathcal{S}_{1}$.

With the above assumptions, one can state the main result of this paper:

\section{Theorem 1}

Under Assumptions 1, 2 and 3, $\mathcal{S}_{1}$ is a RPI set for system (1) subject to the event-switched control input (4).

\section{Proof}

It must now be proved that the set $\mathcal{S}_{1}$ satisfies Definition 1 for system (1) with the event-switched control input (4). The dynamics of this system were described in equation (5), which is recalled here:

$$
x_{k+1}= \begin{cases}A x_{k}+E w_{k} & \text { if } x_{k} \in \mathcal{S}_{2} \\ (A-B K) x_{k}+E w_{k} & \text { otherwise }\end{cases}
$$

Let us assume the existence of an arbitrary state $x_{k}$ in $\mathcal{S}_{1}$. As far as the dynamics of system (5) are concerned, there are two possible cases: 
- $x_{k} \in \mathcal{S}_{2}$, which means that $x_{k+1}$ will also be in $\mathcal{S}_{1}$ because the set $\mathcal{S}_{2}$ meets the condition (9) from Assumption 2.

- $x_{k} \notin \mathcal{S}_{2}$, which means that $x_{k+1}$ will still be in $\mathcal{S}_{1}$ because it is a RPI set for the associated dynamics resulting from Assumption 3.

This proves that $\mathcal{S}_{1}$ is a RPI set for system (5), because for any $x_{0}$ in $\mathcal{S}_{1}, x_{k}$ will be in $\mathcal{S}_{1}$ for all values of $k \in \mathbb{N}$.

Theorem 2

Under Assumptions 1, 2, and 4, system (1) with the event-switched control input (4) is UB within $\mathcal{S}_{1}$.

Proof

It now has to be proved that for any initial state $x_{0}$, there exists $K\left(x_{0}\right) \in \mathbb{N}$ such that $x_{k}$ will be in $\mathcal{S}_{1}$ for all $k \geq K\left(x_{0}\right)$. When dealing with system (5), there are two possible cases:

- $x_{0} \in \mathcal{S}_{2}$ means that $x_{k}$ will join $\mathcal{S}_{1}$ in $K\left(x_{0}\right)=1$ time step resulting from (9).

- $x_{0} \notin \mathcal{S}_{2}$, based on Assumption 4, $x_{k}$ will join $\mathcal{S}_{1}$ in a finite number of time steps, namely $K\left(x_{0}\right)$.

Once $x_{k}$ is in $\mathcal{S}_{1}$, i.e. once $k$ is greater than or equal to $K\left(x_{0}\right)$, there are again two possibilities:

- $x_{k} \in \mathcal{S}_{2}$, which means that $x_{k+1}=A x_{k}+E w_{k}$ will be in $\mathcal{S}_{1}$, for all $k \geq K\left(x_{0}\right)$ because the set $\mathcal{S}_{2}$ satisfies condition (9) based on Assumption 2.

- $x_{k} \notin \mathcal{S}_{2}$, which means that $x_{k+1}=(A-B K) x_{k}+E w_{k}$ will be in $\mathcal{S}_{1}$, for all $k \geq K\left(x_{0}\right)$, based on Assumption 4.

Therefore, there exists $K\left(x_{0}\right)$ such that, for any $x_{0} \in \mathbb{R}^{n}, x_{k}$ will be in $\mathcal{S}_{1}$ for all $k \geq K\left(x_{0}\right)$. This means that system (5) is UB within $\mathcal{S}_{1}$, based on Definition 2.

\section{Remark 4}

These theorems will still hold if $\mathcal{S}_{2}$ is an empty, non-polytopic or non-convex set.

\subsection{Analytic discussion of performances}

In this section, it is assumed that $\mathcal{S}_{1}$ is a RPI set for system (1), $\mathcal{S}_{2}$ is an event-set for system (1) and $\mathcal{S}_{1}$. It is also assumed that the initial state $x_{0}$ of system (1) is in the RPI set $\mathcal{S}_{1}$. In order to assess the performances of the present strategy and compare them with those obtained with a classical approach, an upper bound is calculated for the 2-norm of i) the state $x$, ii) the control input $u$, and iii) the changing rate of the control $\Delta u$. In what follows, these indicators will be denoted $\delta_{x}, \delta_{u}$, and $\delta_{d}$, respectively. In addition, the superscripts ${ }^{e s}$ and ${ }^{t t}$ will refer to the event-switched and timetriggered systems, respectively. Variables without any superscript will be taken to refer to both the time-triggered and the event-switched systems.

3.2.1. Maintaining the state performances Preserving the bound $\delta_{x}$ on the maximal 2-norm of the state results directly from Theorem 1: the fact that the systems (1) under time-triggered control (2) and event-switched control (4) have the same RPI set means that the maximum 2-norm of the state will be bounded by the radius of the smallest 2-norm ball containing the RPI set.

$$
\|x\|_{2} \leq \delta_{x}, \text { with } \delta_{x} \triangleq \min \left\{\gamma \mid \mathcal{S}_{1} \subseteq \mathbb{B}_{2}^{n}(\gamma)\right\}
$$

\subsubsection{Maintaining the control signal performances}

Maintaining the amplitude Maintaining the bound $\delta_{u}$ also results from Theorem 1. In the timetriggered case, the controller (3) is linear, and the control input $u$ is therefore bounded in a set $\mathcal{U}$ which is the projection of the set $\mathcal{S}_{1}$ in the control space by the linear matrix mapping $-K$. In the event-switched case, the non-linear controller (4) can switch between two modes. In one mode, 
when $x \notin \mathcal{S}_{2}$, the control input $u=-K x$ is obviously bounded in the set $\mathcal{U}$. In the other mode, when $x \in \mathcal{S}_{2}$, the control input signal is $0_{m}$, which is in $\mathcal{U}$ because $\mathcal{S}_{1}$ includes the origin, and the mapping between $\mathcal{S}_{1}$ and $\mathcal{U}$ is linear.

$$
u \in \mathcal{U}, \text { with } \mathcal{U} \triangleq-K \cdot \mathcal{S}_{1}
$$

It follows directly that:

$$
\|u\|_{2} \leq \delta_{u}, \text { with } \delta_{u} \triangleq \min \left\{\gamma \mid \mathcal{U} \subseteq \mathbb{B}_{2}^{n}(\gamma)\right\}
$$

Maintaining the changing rate The changing rate of the control input is:

$$
\Delta u_{k}=u_{k+1}-u_{k}
$$

In the case of the time-triggered system, this gives:

$$
\begin{aligned}
& \Delta u_{k}^{t t}=u_{k+1}^{t t}-u_{k}^{t t} \\
& \Delta u_{k}^{t t}=-K x_{k+1}^{t t}-\left(-K x_{k}\right) \\
& \Delta u_{k}^{t t}=-K\left(\left(A_{C L}-I_{n}\right) x_{k}+E w_{k}\right)
\end{aligned}
$$

It follows that:

$$
\begin{aligned}
& \Delta u_{k}^{t t} \in \mathcal{D}^{t t}, \forall k \in \mathbb{N} \\
& \text { with } \mathcal{D}^{t t} \triangleq-K\left(\left(A_{C L}-I_{n}\right) \mathcal{S}_{1} \oplus E \mathcal{W}\right)
\end{aligned}
$$

The bound of the 2-norm of the changing control input rate in the time-triggered system is therefore:

$$
\left\|\Delta u_{k}{ }^{t t}\right\|_{2} \leq \delta_{d}{ }^{t t}, \text { with } \delta_{d}{ }^{t t} \triangleq \min \left\{\gamma \mid \mathcal{D}^{t t} \subseteq \mathbb{B}_{2}^{n}(\gamma)\right\}
$$

In the case of the event-switched system, several possibilities have to be considered:

$$
\begin{aligned}
& u_{k}^{e s}= \begin{cases}0_{m} & \text { if } x_{k} \in \mathcal{S}_{2} \\
-K x_{k} & \text { otherwise }\end{cases} \\
& u_{k+1}^{e s}=\left\{\begin{array}{lllll}
0_{m} & \text { if } & x_{k+1}^{e s} \in \mathcal{S}_{2} & & \\
-K\left(A x_{k}+E w_{k}\right) & \text { if } & x_{k+1}^{e s} \notin \mathcal{S}_{2} & \text { and } & x_{k} \in \mathcal{S}_{2} \\
-K\left(A_{C L} \cdot x_{k}+E w_{k}\right) & \text { if } & x_{k+1}^{e s} \notin \mathcal{S}_{2} & \text { and } & x_{k} \notin \mathcal{S}_{2}
\end{array}\right.
\end{aligned}
$$

Therefore:

$$
\Delta u_{k}^{e s}=u_{k+1}^{e s}-u_{k}^{e s}=\left\{\begin{array}{llll}
0_{m} & \text { if } x_{k+1}^{e s} \in \mathcal{S}_{2} & \text { and } & x_{k} \in \mathcal{S}_{2} \\
K x_{k} & \text { if } x_{k+1}^{e s} \in \mathcal{S}_{2} & \text { and } & x_{k} \notin \mathcal{S}_{2} \\
-K\left(A x_{k}+E w_{k}\right) & \text { if } x_{k+1}^{e s} \notin \mathcal{S}_{2} & \text { and } & x_{k} \in \mathcal{S}_{2} \\
-K\left(\left(A_{C L}-I_{n}\right) x_{k}+E w_{k}\right) & \text { if } x_{k+1}^{e s} \notin \mathcal{S}_{2} & \text { and } & x_{k} \notin \mathcal{S}_{2}
\end{array}\right.
$$

It follows that:

$$
\begin{aligned}
& \Delta u_{k}^{e s} \in \mathcal{D}^{e s}, \forall k \in \mathbb{N} \\
& \text { with } \quad \mathcal{D}^{e s} \triangleq 0_{m} \cup K \cdot \mathcal{S}_{1} \cup-K\left(A \cdot \mathcal{S}_{2} \oplus E \cdot \mathcal{W}\right) \cup \mathcal{D}^{t t}
\end{aligned}
$$

Note that the expression for $\mathcal{D}^{e s}$ can be simplified because the origin is included in $\mathcal{S}_{1}$ :

$$
\mathcal{D}^{e s}=K \cdot \mathcal{S}_{1} \cup-K \cdot\left(A \cdot \mathcal{S}_{2} \oplus E \cdot \mathcal{W}\right) \cup \mathcal{D}^{t t}
$$

and since $\left(A \cdot \mathcal{S}_{2} \oplus E \cdot \mathcal{W}\right)$ is included in $\mathcal{S}_{1}$, in view of condition (9), this means that $\min \{\gamma \mid-$ $\left.K \cdot\left(A \cdot \mathcal{S}_{2} \oplus E \cdot \mathcal{W}\right) \subseteq \mathbb{B}_{2}^{n}(\gamma)\right\} \leq \min \left\{\gamma \mid \mathcal{D}^{t t} \subseteq \mathbb{B}_{2}^{n}(\gamma)\right\}$. The bound of the 2-norm of the changing 
control input rate in the event-switched system is therefore:

$$
\left\|\Delta u_{k}^{e s}\right\|_{2} \leq \delta_{d}^{e s}, \quad \text { with } \quad \delta_{d}^{e s} \triangleq \max \left(\delta_{d}^{t t}, \min \left\{\gamma \mid K \cdot \mathcal{S}_{1} \subseteq \mathbb{B}_{2}^{n}(\gamma)\right\}\right)
$$

Therefore, if $\delta_{d}^{t t} \geq\left\|K \cdot \mathcal{S}_{1}\right\|_{2}$, then $\delta_{d}^{e s}=\delta_{d}^{t t}$. In other words, the 2-norm of the changing control input rate has the same least upper bound in both the time-triggered and the event-switched system if $\delta_{d}^{t t} \geq \min \left\{\gamma \mid K \cdot \mathcal{S}_{1} \subseteq \mathbb{B}_{2}^{n}(\gamma)\right\}$

\section{Remark 5}

If $\mathcal{S}_{1}$ is the mRPI set and the event-set $\mathcal{S}_{2}$ is the largest set $\mathcal{S}_{2}^{\star}$ satisfying condition (9), then $\left(A \cdot \mathcal{S}_{2} \oplus E \cdot \mathcal{W}\right)=\mathcal{S}_{1}$ and the set $\mathcal{D}^{e s}$ becomes:

$$
\mathcal{D}^{e s}=K \cdot \mathcal{S}_{1} \cup-K \cdot \mathcal{S}_{1} \cup \mathcal{D}^{e s}
$$

In addition, if the bound of the disturbance is symmetric, then $\mathcal{S}_{1}$ will also be symmetric, and therefore:

$$
\mathcal{D}^{e s}=K \cdot \mathcal{S}_{1} \cup \mathcal{D}^{t t}
$$

\subsection{Observer based approach}

The above approach involves checking the value of the state vector at each time step $k$ in order to check whether or not the state of the system belong to the event-set $\mathcal{S}_{2}$. Depending on the result, it is then decided whether or not to apply a control signal without detracting from the performances of the scheme. However, it is assumed throughout this strategy that all the state information is available. When the state of the system (1) cannot be completely measured and only the output $y_{k}$ is available, the complete system is modeled as follows :

$$
\begin{aligned}
x_{k+1} & =A x_{k}+B u_{k}+E w_{k} \\
y_{k} & =C x_{k}
\end{aligned}
$$

One possible solution here is to construct a Luenberger observer (assuming that the matrix pair $(A, C)$ is observable):

$$
\hat{x}_{k+1}=A \hat{x}_{k}+B u_{k}+L\left[y_{k}-C \hat{x}_{k}\right]
$$

where $\hat{x}$ is the observed state, and $L$ the observer gain. The challenge here is then to find how to use the event-switched method presented above in the case of system (36)-(37) ensuring accurate performances.

The observation error $e_{k}=x_{k}-\hat{x}_{k}$ has the following dynamics:

$$
e_{k+1}=(A-L C) e_{k}+E w_{k}
$$

Let $\mathcal{S}_{e}$ denote a RPI set in the observation error space, it is a set in which the observation error will be bounded once the observer has converged. The observed state actually has the following dynamics:

$$
\hat{x}_{k+1}=(A-B K) \hat{x}_{k}-L C e_{k}
$$

where the term $e_{k}$ denotes a disturbance bounded in a RPI set $\mathcal{S}_{e}$. A RPI set for system (39) can be computed with the method presented in Section 2.1, and will be denoted $\hat{\mathcal{S}}_{1}$. Lastly, an event-set $\mathcal{S}_{2}$ can be found as described in Section 2.2 in the observed state space, where the complete system with an observer can be in an open-loop configuration when the observed state is in $\mathcal{S}_{2}$, while still ensuring that $\hat{\mathcal{S}}_{1}$ is a RPI set for system (39).

\subsection{Proposed algorithm}

In the present paper, a new approach to designing event-switched control is presented. With the present event-switched control approach, the system does not have to be controlled between events, and efficient performances can still be obtained even in the presence of bounded 


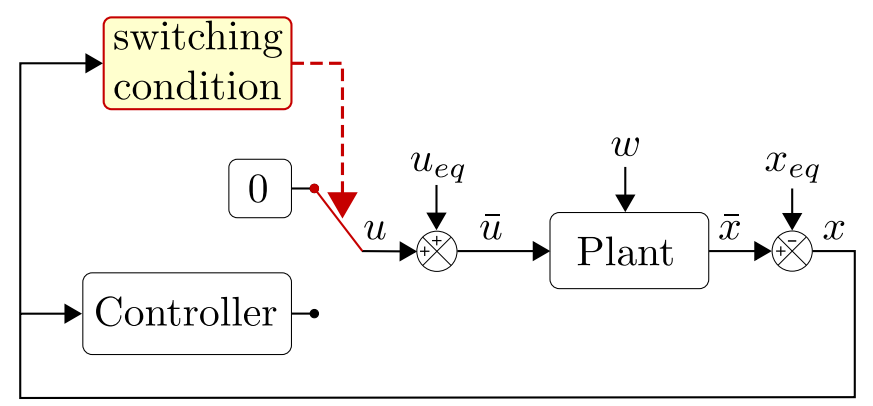

Figure 1. Event-switched control strategy for a plant with an exogenous disturbance $w$. The switching condition is used to determine whether an action has to be performed by the controller or not.

disturbances. A switching condition is checked periodically in order to determine whether ot not a dynamic control input has to be applied. Fig. 1 presents the overall control strategy.

The first step in the present method consists in finding the nominal performances of the controlled system subjected to bounded disturbances. The nominal performances correspond to a RPI set for the system. We take $\mathcal{S}_{1}$ to denote this set. Note that as $\mathcal{S}_{1}$ is a RPI set, once the state has entered this set, it will remain there. In order to be less conservative, $\mathcal{S}_{1}$ can be approximated as the mRPI set. The second step consists in finding an event-set $\mathcal{S}_{2}$. When the state of the system is located inside $\mathcal{S}_{2}$, the control input will switch off and the system will run in the (possibly unstable) open-loop mode. Whenever the state no longer belongs to $\mathcal{S}_{2}$, the control input switches on again. Fig. 2 illustrates the whole process in the case of a 2-dimensional system (in order to simplify the spatial representation). It is worth noting that the event-set $\mathcal{S}_{2}$ is not included in $\mathcal{S}_{1}$ in this particular example, $\mathcal{S}_{2} \backslash \mathcal{S}_{1}$ can only be reached one time step before joining $\mathcal{S}_{1}$. Three different cases arise here:

1: When the state does not belong to both the nominal performance set $\mathcal{S}_{1}$ and the event-set $\mathcal{S}_{2}$, the linear control input is applied to the system and the state will join either $\mathcal{S}_{1}$ or $\mathcal{S}_{2}$ after a finite number of time steps.

2: When the state belongs to $\mathcal{S}_{1}$ but not to $\mathcal{S}_{2}$, the linear control input is applied. At the next time step, the state will remain in $\mathcal{S}_{1}$ and can join $\mathcal{S}_{2} \cap \mathcal{S}_{1}$.

3: When the state belongs to $\mathcal{S}_{2}$, the control input is switched off, and only the equilibrium value $u_{e q}$ is applied to the control input (or $0_{m}$ in the null stabilization case). Due to the mathematical properties of $\mathcal{S}_{2}$, the state will belong to $\mathcal{S}_{1}$ at the next time step (and can also continue to belong to $\mathcal{S}_{2} \cap \mathcal{S}_{1}$ ).

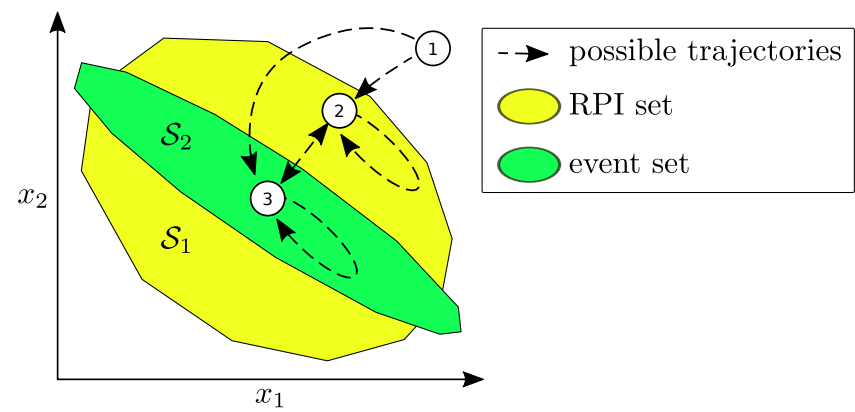

Figure 2. Geometrical interpretation of the event-switched control strategy illustrating three different possible state trajectories of the event-switched system. $\mathcal{S}_{1}$ is a RPI set and $\mathcal{S}_{2}$ is the event-set. It is worth noting that $\mathcal{S}_{2}$ is not included in $\mathcal{S}_{1}$.

Lastly, designing an event-switched control strategy with the approach presented here can be done using a systematic method. Given any stabilizable system (1): 
- Find a linear state-feedback $K$ such that the eigenvalues of $A-B K$ are inside the unit circle.

- Compute an approximation for the mRPI set of the system. See [33] for an efficient method.

- Compute the event-set $\mathcal{S}_{2}^{\star}$ as explained in Section 2.2.

- Take any set $\mathcal{S}_{2} \subseteq \mathcal{S}_{2}^{\star}$ as an event-set.

All these steps are performed to compute an event condition which ensures efficient performances. This event condition can be checked online as described in the Algorithm 1.

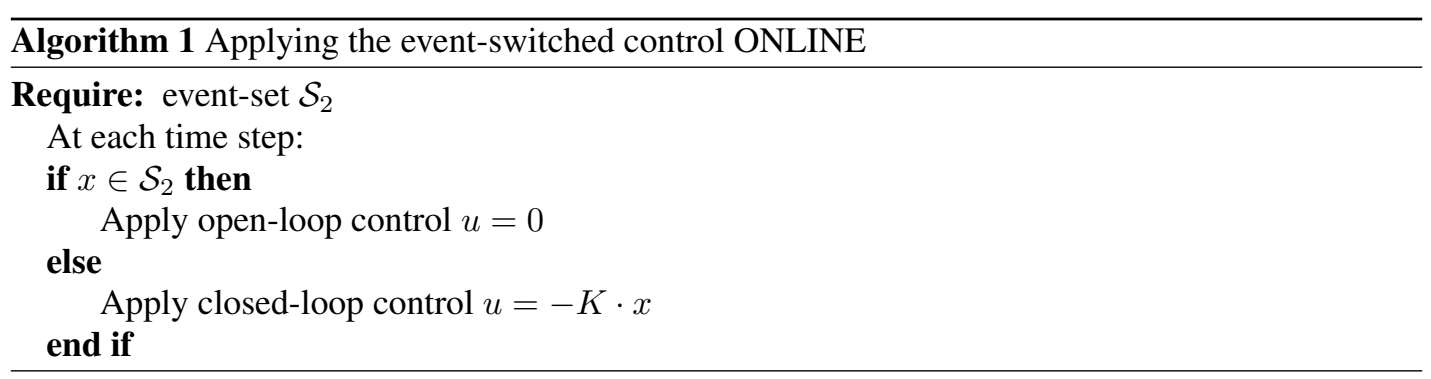

We recall that the event-set $\mathcal{S}_{2}$ is computed offline, then the inclusion condition checked at each sampling time is very simple to implement on practical applications. The computational effort required for this step depends on the dimension and the topology of the event-set. If the eventset is a polyhedron, the complexity is related to the number of vertices describing the set. To be more precise, it will consist in verifying as many inequalities as the polyhedron has faces. If the set is an ellipsoid, then the complexity is quadratic in the order of the system. The use of an ellipsoidal event-set is discussed in Section 2.3, the results obtained with an implementation of this method has been illustrated in Section 4.3.

The cost of the offline computation is highly related to the considered system. The time required for this computation is given in Section 4 for a given example.

\section{SIMULATION}

In this last section, the approach presented here is applied to the following academic example:

$$
x_{k+1}=\underbrace{\left[\begin{array}{cc}
1 & 0.65 \\
0 & 1
\end{array}\right]}_{A} x_{k}+\underbrace{\left[\begin{array}{c}
0.211 \\
0.65
\end{array}\right]}_{B}\left(u_{k}+w_{k}\right)
$$

Note that in this particular case, the disturbance matrix $E$ is taken to be equal to $B$.

A stabilizing static state-feedback gain was found using the Linear Quadratic (LQ) optimal control method with identity weighting matrices of suitable dimension.

$$
u_{k}=-\underbrace{\left[\begin{array}{ll}
0.575 & 1.217
\end{array}\right]}_{K} x_{k}
$$

The dynamics of the linear discrete time-triggered closed-loop system are therefore:

$$
\begin{aligned}
& x_{k+1}=A_{C L} x_{k}+B w_{k} \\
& \text { with } A_{C L} \triangleq A-B K=\left[\begin{array}{cc}
0.879 & 0.393 \\
-0.374 & 0.209
\end{array}\right]
\end{aligned}
$$

It can be noted that the poles $(0.54 \pm 0.19 \cdot i)$ of the closed-loop system are located inside the unit circle. The system is therefore stable, whereas the open-loop system is unstable. During the simulation, $w$ will be bounded in the set $\mathcal{W}=[-1 ;+1]$.

Both the classical time-triggered closed-loop system (42) and system (40) subjected to the switched control input (4) are simulated in parallel, in order to compare the performances of these 
two systems when the same disturbance is applied. The results of the simulation are presented in Fig. 3 during the first 10 time steps (in order to show the trajectory of the system clearly). The nominal performance set $\mathcal{S}_{1}$ is an approximation of the mRPI set obtained via the algorithm given in [34]. The set $\mathcal{S}_{2}^{\star}$ is obtained as described in Section 2.1. In our implementation using MATLAB and the MPT toolbox [41], the time required to compute these two sets was approximately 0.8 seconds. These sets, as well as the system trajectories, are plotted in Fig. 3a. The control inputs are also presented with respect to the time steps in Fig. 3b. It can be seen from Fig. 3a that the control signal is 0 when the system trajectory is in the set $\mathcal{S}_{2}^{\star}$ at time steps 3, 4, 5, 7 and 8 .

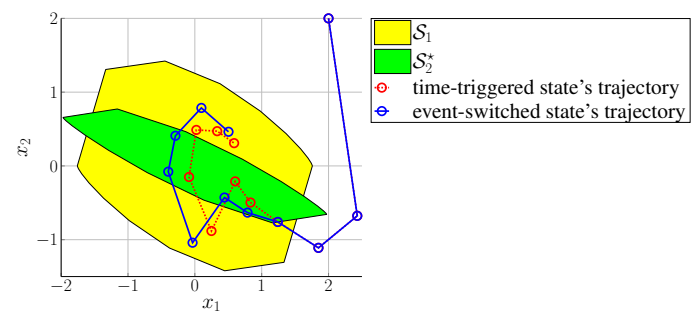

(a) State trajectories.

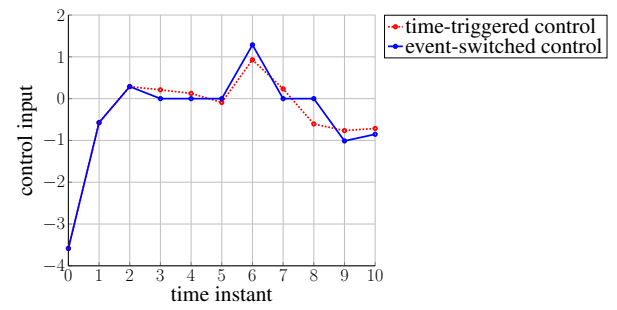

(b) Control input.

Figure 3. Simulation of the time-triggered system (in red) and the event-switched system (in blue) with the same disturbances and initial condition $\left(x_{1}=x_{2}=2\right)$ during 10 time steps. When the state of the eventswitched system is in $\mathcal{S}_{2}^{\star}$ (green set), open-loop control is applied. The set $\mathcal{S}_{1}$ (yellow) describes a portion of the state space in which the state is bounded once it has entered this set in the case of both the time-triggered and the event-switched system.

In order to test the accuracy of the mRPI set approximation, the discrete time system (42) was simulated with the disturbance reaching the bounds. The results obtained, which are presented in Fig. 4, clearly show that the mRPI set approximation is satisfactory, since the state $x$ still belong to the set $\mathcal{S}_{1}$ with the worst disturbance scenario, and is located very near the vertices.

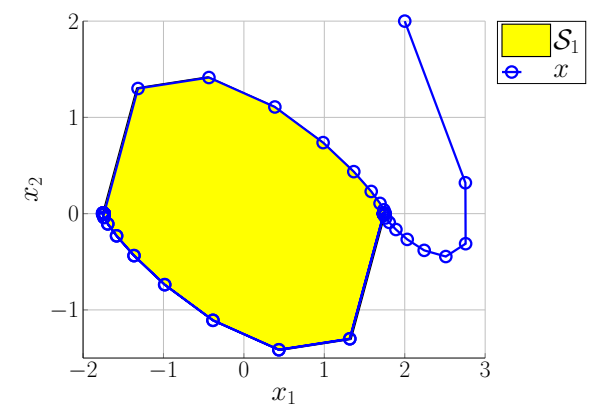

(a) State trajectories.

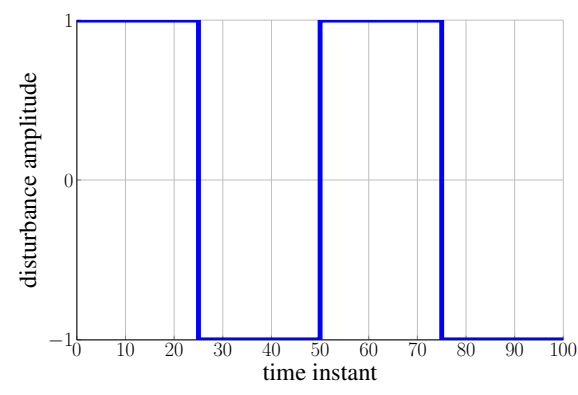

(b) Worst case disturbance.

Figure 4. Worst disturbance scenario for mRPI approximation $\left(\mathcal{S}_{1}\right)$ verification.

In the rest of this section, the simulation was run for $N=10^{7}$ periods of time in order to have a sufficiently good distribution of the disturbances and reliable performance indexes, as described in the next section.

\subsection{Performance indexes}

The following performance indexes were used to compare the efficiency of the process:

- The maximum 2-norm of the state: $\max \left(\|x\|_{2}\right)$, which is bounded by $\delta_{x}$ in (17);

- The maximum 2-norm of the control input: $\max \left(\|u\|_{2}\right)$, which is bounded by $\delta_{u}$ in (19);

- The maximum 2-norm of the rate of variation of the control input: $\max \left(\|\Delta u\|_{2}\right)$, which is bounded by $\delta_{d}$ in (24) and (33); 
- The percentage time spent in the closed loop: $\% c$ (this index also gives the rate of data bus use between the controller and the actuator in the case of networked control systems). The maximum value is $100 \%$ (in the case of time-triggered control), and the aim is to reduce this percentage with the event-switched control strategy presented here.

In this particular example, the bound $\delta_{d}$ on $\|\Delta u\|_{2}$ is the same in the case of both the time-triggered and the event-switched system because $\delta_{d}^{t t} \geq \min \left\{\gamma \mid K \cdot \mathcal{S}_{1} \subseteq \mathbb{B}_{2}^{n}(\gamma)\right\}$, refer to Section 3.2.2 for details.

The bound values are given in Table I. The performance indexes obtained in the simulations are given in Tables II-III and discussed below.

Table I. Theoretical bounds on performance indexes

\begin{tabular}{|c|c|c|}
\hline$\delta_{x}$ & $\delta_{u}$ & $\delta_{d}$ \\
\hline 1.87 & 1.57 & 1.97 \\
\hline
\end{tabular}

Table II. Performance indexes obtained with uniformly distributed disturbances

\begin{tabular}{|l|c|c|c|c|}
\hline & $\% \mathrm{c}$ & $\max \left(\|x\|_{2}\right) \leq \delta_{x}$ & $\max \left(\|u\|_{2}\right) \leq \delta_{u}$ & $\max \left(\|\Delta U\|_{2}\right) \leq \delta_{d}$ \\
\hline Time-triggered strategy & 100 & 1.69 & 1.48 & 1.92 \\
\hline Event-switching strategy with $\mathcal{S}_{2}^{\star}$ & 48.31 & 1.71 & 1.57 & 1.95 \\
\hline Event-switching strategy with $\mathcal{E}^{\star}$ & 49.66 & 1.70 & 1.52 & 1.95 \\
\hline
\end{tabular}

Table III. Performance indexes obtained with truncated normally distributed disturbances

\begin{tabular}{|l|c|c|c|c|}
\hline & $\% \mathrm{c}$ & $\max \left(\|x\|_{2}\right) \leq \delta_{x}$ & $\max \left(\|u\|_{2}\right) \leq \delta_{u}$ & $\max \left(\|\Delta U\|_{2}\right) \leq \delta_{d}$ \\
\hline Time-triggered strategy & 100 & 1.58 & 1.42 & 1.79 \\
\hline Event-switching strategy with $\mathcal{S}_{2}^{\star}$ & 39.62 & 1.64 & 1.55 & 1.90 \\
\hline Event-switching strategy with $\mathcal{E}^{\star}$ & 39.44 & 1.64 & 1.52 & 1.90 \\
\hline
\end{tabular}

\subsection{Results}

Fig. 5 shows the results of the simulation when a uniformly distributed disturbance has been applied. The simulated reachable set denoted $\mathcal{S}_{x}$ after $10^{7}$ time steps starting at the origin in the case of the time-triggered system can be seen in Fig. 5a, and in the case of the event-switched system, in Fig. 5b. $\mathcal{S}_{x}$ is the convex hull where the state remains during the simulation. It can also be noted from Fig. 5b that the state continues to stay in $\mathcal{S}_{1}$ throughout the simulation, as $\mathcal{S}_{x}$ is included in $\mathcal{S}_{1}$. This is the expected behavior of the present event-switched control method as $\mathcal{S}_{1}$ is a RPI set for both the time-triggered and the event-switched systems.

The performance indexes introduced in Section 4.1 are presented in Tables II and III in the case of truncated normal and uniform disturbance distributions, respectively. The results show that the 2-norms of the state are bounded as predicted by $\delta_{x}$, as explained in Section 3.2.1. This can also be seen to occur with a geometrical interpretation, as $\mathcal{S}_{x}$ is included in $\mathcal{S}_{1}$. The control input and the changing rate of the control input are also bounded by $\delta_{u}$ and $\delta_{d}$, respectively. However, it can be seen that in the event-switched case, the reachable set $\mathcal{S}_{x}$ is slightly larger than that obtained using the time-triggered control. This difference may be attributable to the random nature of the disturbances and their impact on the bound of the reachable set $\mathcal{S}_{x}$ : see for instance probabilistic invariant sets for linear systems [42]. It is recalled, however, that the state trajectories occurring in the worst disturbance scenario, which are shown in Fig. 4a, belong to the boundaries of the set $\mathcal{S}_{1}$ in both cases.

It is also worth considering the frequency domain of the state and the control input in the above simulation. Fig. 6 shows the power spectral density (PSD) of the control input $u$, and the states 


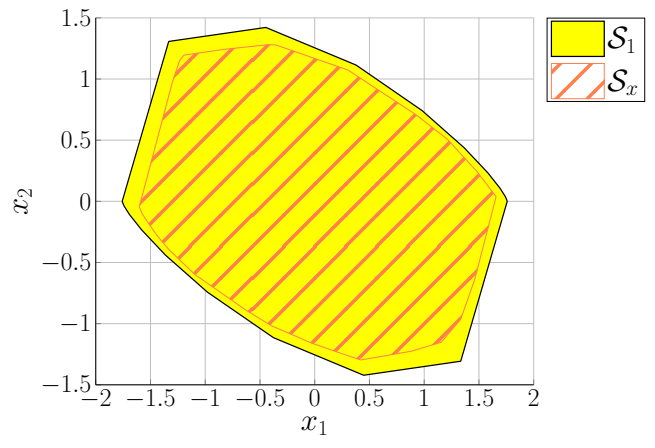

(a) Time-triggered system's state.

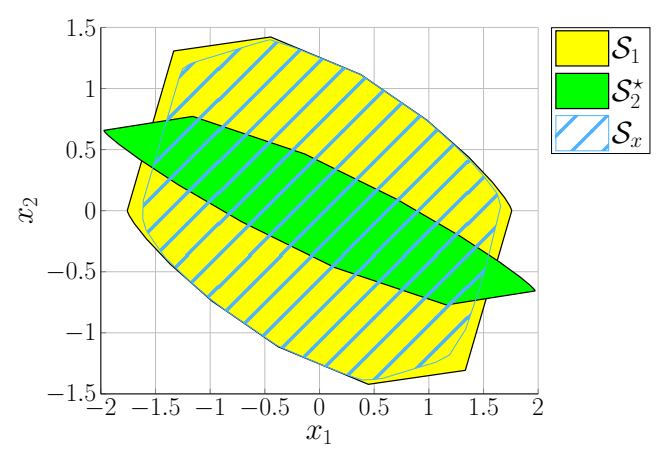

(b) Event-switched system's state.

Figure 5. Comparison between time-triggered and event-switched systems subjected to the same uniformly distributed disturbances. $\mathcal{S}_{1}$ is an approximation for the mRPI set computed offline, as mentioned in Section 2.1, and $\mathcal{S}_{x}$ is the reachable set from the origin after $10^{7}$ periods of time.

$x_{1}$ and $x_{2}$ (where $x^{T}=\left[\begin{array}{ll}x_{1} & x_{2}\end{array}\right]$ ). It can be noted that the magnitude of these values is generally greater in the case of the event-switched control system than with the classical approach. However, the overall shape is similar in both cases. It can be seen from Fig. 6a that the magnitude of the control input is greater at high frequencies in the case of the event-switched method. But in $x_{1}$ in Fig. 6b and $x_{2}$ in Fig. 6c, lower values were obtained at high frequencies in the case of the event-switched strategy. This is a particularly noteworthy result of the simulation: applying unstable dynamics to some events can result in less energy in the high frequency domain of the state.

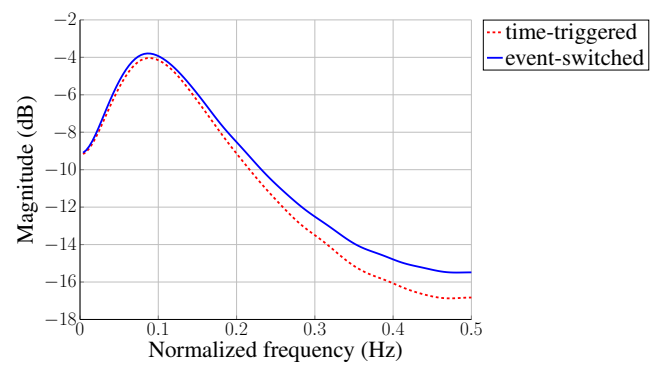

(a) Power spectral density of the control input.

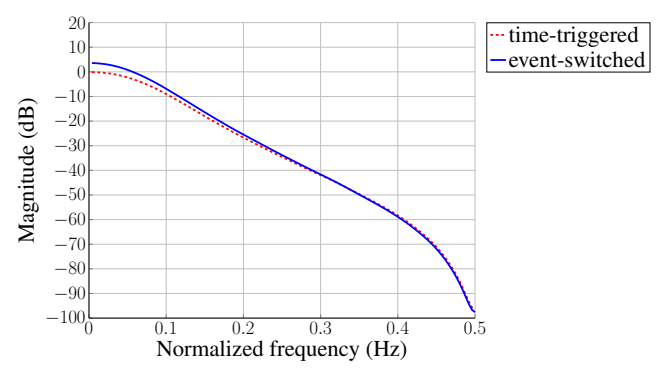

(b) Power spectral density of $x_{1}$.

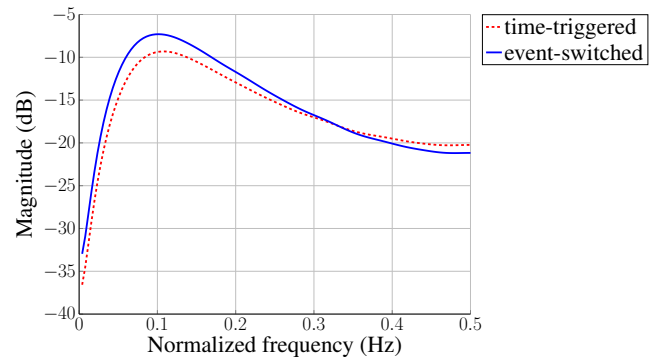

(c) Power spectral density of $x_{2}$.

Figure 6. Power spectral density comparison between time-triggered and event-switched systems.

\subsection{Reducing computation complexity}

To use the switching mechanism (4), one has to check at each time instant whether the state belongs to $\mathcal{S}_{2}^{\star}=\left\{x \in \mathbb{R}^{n}: R x \leq Q\right\}$ with $R \in \mathbb{R}^{f \times n}$ and $Q \in \mathbb{R}^{f}$. For this purpose, one only needs to check whether the state meets the condition imposed in the set definition: $R x \leq Q$. In the above example, the set $\mathcal{S}_{2}^{\star}$ is a polyhedron defined by the intersection between 20 half-spaces, which 
means calculating $2 \times 20=40$ multiplications, 20 additions and checking 20 inequalities at each time step.

To simplify the control algorithm, another event-set can be defined inside $\mathcal{S}_{2}^{\star}$, such as the largest ellipse inscribed in $\mathcal{S}_{2}^{\star}$. In the above example, this would reduce the number of operations to 6 multiplications, 3 additions and 1 inequality per time step. However, this would mean that the resulting event-switched system would switch to open-loop dynamics less frequently as the volume of the event-set would be smaller.

Computing the largest ellipsoid in a polyhedron is a convex optimization problem, as stated in [43, Section 8.4.1]. Let us define the ellipse $\mathcal{E}^{\star}$ as follows:

$$
\mathcal{E}^{\star} \triangleq\{T x+d \mid\|x\| \leq 1\}, \text { with } T \text { a symmetric matrix }
$$

In order to maximize the volume of $\mathcal{E}^{\star}$, the constraint which has to be met is that every element of $\mathcal{E}^{\star}$ has to satisfy the inequality defining $\mathcal{S}_{2}^{\star}$. This could be written as follows:

$$
\begin{array}{ll}
\underset{T, d}{\operatorname{maximize}} & \log (\operatorname{det} T) \\
\text { subject to } & \|T R\|+R d \leq Q, \text { and } T \text { symmetric }
\end{array}
$$

$\mathcal{E}^{\star}$ can also be defined in a more popular form than (43), as follows:

$$
\mathcal{E}^{\star} \triangleq\left\{(x-d)^{T} S(x-d) \leq 1\right\}, \text { with } S=T^{-2}
$$

The event-set $\mathcal{E}^{\star}$ thus obtained is presented in Fig. 7. A simulation was run with the event-set denoted $\mathcal{E}^{\star}$, and the results obtained are presented in Tables II and III. As was to be expected, the total amount of time during which the plant was allowed to be in the open-loop mode was shorter than with the event-set $\mathcal{S}_{2}^{\star}$. However, the difference amounted to only about $1 \%$, which means that the advantages of the present method were not greatly decreased. The performance indexes obtained were are almost the same as in Section 4.2. Choosing the set where the system is allowed to be in the open loop mode therefore amounts to making a trade-off between the complexity of the event conditions and the performances of the process.

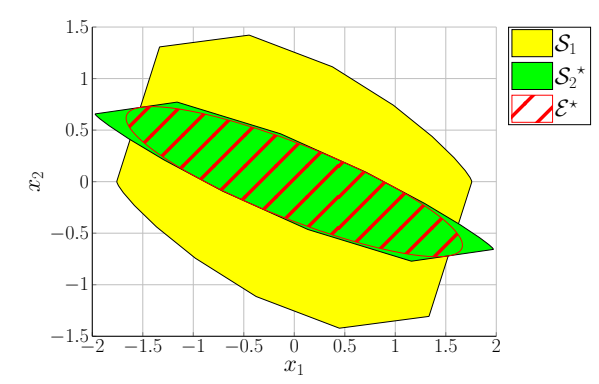

Figure 7. The set $\mathcal{E}^{\star}$ is the maximum volume ellipse included in $\mathcal{S}_{2}^{\star}$.

\section{CONCLUSIONS AND LINES OF FUTURE RESEARCH}

The novel approach to designing event-switched systems presented in this paper is applicable to discrete linear time-invariant systems subjected to bounded disturbances. The main advantage of this method is to ensure the existence of the same RPI sets as those occurring in the equivalent classical time-triggered systems, while allowing the system to use an open-loop controller if the state belongs to an event-set. A method is described for computing this event-set, assuming that a RPI set is already known. This method ensures upper bound of the 2-norm of the state, the control input and the changing control input rate. The main contribution of this paper is to provide an event 
mechanism and a systematic method to compute the event condition with specifying the required performances a priori.

Simulations performed on an example showed that the method presented here makes it possible to use an open-loop controller more than half of the time, while some performances are maintained. Although a specific event condition has to be checked at each time step in the original method, another condition can also be used, which gives almost similar results while simplifying the test procedure.

It is proposed in future studies to extend this approach to continuous systems by including intersample behavior. Designing an observer with the present approach would also be worth studying in order to limit the number of communications required between a sensor measuring the output of the system and the controller.

\section{ACKNOWLEDGEMENT}

This research was partly supported by the LabEx PERSYVAL-Lab (ANR-11-LABX-0025).

\section{REFERENCES}

1. Ogata K. Discrete-time Control Systems. Prentice-Hall, Inc.: Upper Saddle River, NJ, USA, 1987.

2. Åström KJ, Bernhardsson B. Comparison of periodic and event based sampling for first-order stochastic systems. Proceedings of the 14th IFAC World congress, vol. 11, 1999; 301-306.

3. Hsu P, Sastry S. The effect of discretized feedback in a closed loop system. Proceedings of the 26th IEEE Conference on Decision and Control, vol. 26, 1987; 1518-1523, doi:10.1109/CDC.1987.272670.

4. Nešić D, Teel AR. A framework for stabilization of nonlinear sampled-data systems based on their approximate discrete-time models. IEEE Transactions on Automatic Control jul 2004; 49(7):1103-1122, doi:10.1109/TAC.2004. 831175.

5. Nešić D, Grüne L. Lyapunov-based continuous-time nonlinear controller redesign for sampled-data implementation. Automatica 2005; 41(7):1143-1156, doi:10.1016/j.automatica.2005.03.001.

6. Årzén KE. A simple event-based PID controller. Proceedings of the 14th IFAC World congress, 1999.

7. Durand S, Marchand N. Further results on event-based PID controller. European Control Conference, 2009; 1979 1984.

8. Sandee JH, Heemels WPMH, van den Bosch PPJ. Event-driven control as an opportunity in the multidisciplinary development of embedded controllers. American Control Conference, vol. 3, 2005; 1776-1781, doi:10.1109/ACC. 2005.1470225.

9. Sánchez J, Guarnes MA, Dormido S. On the application of different event-based sampling strategies to the control of a simple industrial process. Sensors 2009; 9(9):6795, doi:10.3390/s90906795.

10. Åström KJ, Bernhardsson BM. Comparison of Riemann and Lebesgue sampling for first order stochastic systems. Proceedings of the 41st IEEE Conference on Decision and Control, vol. 2, 2002; 2011-2016, doi:10.1109/CDC. 2002.1184824.

11. Heemels WPMH, Sandee JH, Bosch PPJVD. Analysis of event-driven controllers for linear systems. International Journal of Control Apr 2008; 81(4):571-590, doi:10.1080/00207170701506919.

12. Lunze J, Lehmann D. A state-feedback approach to event-based control. Automatica 2010; 46(1):211-215, doi: 10.1016/j.automatica.2009.10.035.

13. Eqtami A, Dimarogonas DV, Kyriakopoulos KJ. Event-triggered control for discrete-time systems. American Control Conference, 2010; 4719-4724, doi:10.1109/ACC.2010.5531089.

14. Durand S. Event-based stabilization of linear system with communication delays in the measurements. American Control Conference, 2013; 152-157, doi:10.1109/ACC.2013.6579829.

15. Velasco M, Martí P, Bini E. On Lyapunov sampling for event-driven controllers. Proceedings of the 48th IEEE Conference on Decision and Control, held jointly with the 28th Chinese Control Conference, 2009; 6238-6243, doi:10.1109/CDC.2009.5400541.

16. Tabuada P. Event-triggered real-time scheduling of stabilizing control tasks. IEEE Transactions on Automatic Control Sep 2007; 52(9):1680-1685, doi:10.1109/TAC.2007.904277.

17. Anta A, Tabuada P. To sample or not to sample: Self-triggered control for nonlinear systems. IEEE Transactions on Automatic Control Sep 2010; 55(9):2030-2042, doi:10.1109/TAC.2010.2042980.

18. Marchand N, Durand S, Guerrero Castellanos F. A general formula for event-based stabilization of nonlinear systems. IEEE Transactions on Automatic Control 2013; 58(5):1332-1337, doi:10.1109/TAC.2012.2225493.

19. Durand S, Marchand N, Guerrero Castellanos F. Event-based Stabilization of Nonlinear Time-Delay Systems. Proceedings of the 19th IFAC World Congress, vol. 47, 2014; 6953-6958, doi:10.3182/20140824-6-ZA-1003. 01941.

20. Seuret A, Prieur C, Marchand N. Stability of nonlinear systems by means of event-triggered sampling algorithms. Journal of Mathematical Control and Information 2014; 31(3):415-433, doi:10.1093/imamci/dnt018.

21. Espitia N, Girard A, Marchand N, Prieur C. Event-based control of linear hyperbolic systems of conservation laws. Automatica 2016; 70:275-287, doi:10.1016/j.automatica.2016.04.009. 
22. Dimarogonas DV, Frazzoli E, Johansson KH. Distributed event-triggered control for multi-agent systems. IEEE Transactions on Automatic Control May 2012; 57(5):1291-1297, doi:10.1109/TAC.2011.2174666.

23. Seyboth GS, Dimarogonas DV, Johansson KH. Event-based broadcasting for multi-agent average consensus. Automatica 2013; 49(1):245 - 252, doi:10.1016/j.automatica.2012.08.042.

24. Fan Y, Feng G, Wang Y, Song C. Distributed event-triggered control of multi-agent systems with combinational measurements. Automatica 2013; 49(2):671 - 675, doi:10.1016/j.automatica.2012.11.010.

25. Xu W, Ho DWC, Zhong J, Chen B. Distributed edge event-triggered consensus protocol of multi-agent systems with communication buffer. International Journal of Robust and Nonlinear Control 2016; doi:10.1002/rnc.3582.

26. Xu W, Ho DWC. Clustered event-triggered consensus analysis: An impulsive framework. IEEE Transactions on Industrial Electronics 2016; doi:10.1109/TIE.2016.2584009.

27. Heemels W, Donkers M, Teel A. Periodic event-triggered control for linear systems. IEEE Transactions on Automatic Control Apr 2013; 58(4):847-861, doi:10.1109/TAC.2012.2220443.

28. LaSalle J, Lefschetz S. Stability by Liapunov's direct method: with applications. Mathematics in science and engineering, Academic Press, 1961.

29. Johannesson E, Henningsson T, Cervin A. Sporadic control of first-order linear stochastic systems. Proceedings of the 10th International Conference on Hybrid Systems: Computation and Control, vol. 4416, Springer, 2007; 301-314, doi:10.1007/978-3-540-71493-4\_25.

30. Blanchini F. Ultimate boundedness control for uncertain discrete-time systems via set-induced Lyapunov functions. IEEE Transactions on Automatic Control Feb 1994; 39(2):428-433, doi:10.1109/9.272351.

31. Blanchini F. Survey paper: Set invariance in control. Automatica 1999; 35(11):1747-1767, doi:10.1016/ S0005-1098(99)00113-2.

32. Grüne L, Jerg S, Junge O, Lehmann D, Lunze J, Müller F, Post M. Two complementary approaches to event-based control. Automatisierungstechnik Apr 2010; 58(4):173-182, doi:10.1524/auto.2010.0832.

33. Rakovic SV, Kerrigan EC, Kouramas KI, Mayne DQ. Invariant approximations of the minimal robust positively invariant set. IEEE Transactions on Automatic Control Mar 2005; 50(3):406-410, doi:10.1109/TAC.2005.843854.

34. Kolmanovsky I, Gilbert EG. Theory and computation of disturbance invariant sets for discrete-time linear systems. Mathematical Problems in Engineering 1998; 4(4):317-367, doi:10.1155/S1024123X98000866.

35. Kofman EJ, Haimovich H, Seron MM. A systematic method to obtain ultimate bounds for perturbed systems. International Journal of Control Feb 2007; 80(2):167-178.

36. Seron MM, Zhuo XW, De Doná JA, Martinez JJ. Multisensor switching control strategy with fault tolerance guarantees. Automatica 2008; 44(1):88-97, doi:10.1016/j.automatica.2007.05.024.

37. Martinez JJ, Seron MM, De Doná JA. Multi-sensor longitudinal control with fault tolerant guarantees. European Control Conference, Budapest - Hungary, 2009; 4235-4240.

38. Haimovich H, Kofman E, Seron MM. Analysis and improvements of a systematic componentwise ultimate-bound computation method. Proceedings of the 17th IFAC World Congress, Seoul - South Korea, 2008.

39. Martinez JJ. Minimal RPI sets computation for polytopic systems using the bounded-real lemma and a new shrinking procedure. 1st IFAC Workshop on Linear Parameter Varying systems (IFAC LPVS), vol. 48, 2015; 182 187, doi:10.1016/j.ifacol.2015.11.134.

40. Olaru S, De Doná JA, Seron MM, Stoican F. Positive invariant sets for fault tolerant multisensor control schemes. International Journal of Control 2010; 83(12):2622-2640, doi:10.1080/00207179.2010.535215.

41. Herceg M, Kvasnica M, Jones CN, Morari M. Multi-Parametric Toolbox 3.0. Proceedings of the European Control Conference, Zürich - Switzerland, 2013; 502-510. http: / / control . ee. ethz . ch / mpt.

42. Kofman E, De Doná JA, Seron MM. Probabilistic set invariance and ultimate boundedness. Automatica 2012; 48(10):2670-2676, doi:10.1016/j.automatica.2012.06.074.

43. Boyd S, Vandenberghe L. Convex Optimization. Cambridge University Press: New York, NY, USA, 2004. 